\title{
Load Shedding Scheme with Underfrequency and Undervoltage Corrective Actions to Supply High Priority Loads in Islanded Microgrids
}

\author{
B. de Nadai N., ${ }^{1, *}$, A. C. Zambroni de Souza ${ }^{1}$, J. G. de Carvalho Costa ${ }^{1}$, and Miguel Castilla ${ }^{2}$ \\ ${ }^{1}$ Electrical Systems Institute - Federal University of Itajubá, Itajubá, Brazil \\ ${ }^{2}$ Department of Electronic Engineering, Technical University of Catalonia, Vilanova i la Geltru, Spain \\ "nadaibruno@gmail.com
}

\begin{abstract}
In the last few years, microgrids have become a potential solution to improve reliability and security in the supply of electricity to power systems. When a microgrid changes its operation mode from grid-connected to islanded, particular attention must be paid by the operation services. In the islanded mode, the microgrids' frequency and voltage may reach undesirable values, harming the security and quality of the operation. This paper aims to propose global and local strategies of load shedding to preserve the energy supply to high priority loads within quality standards. The focus of this paper is to consider a microgrid operating only with primary control. In this sense, a proper microgrid time-continuous load flow is applied, considering the constant verification of the frequency and the voltage of all buses. Finally, a Monte Carlo Simulation is used to validate the proposal presented and to give some indices that quantify the load shed in each period. The results show the superior performance of the proposed strategy compared to a state-of-the-art load shedding solution that does not consider the priority of loads.
\end{abstract}

1. Nomenclature

$$
\begin{aligned}
& \text { 1.1. Symbols } \\
& f \text { : System's frequency; } \\
& V_{i} \text { : Terminal voltage at bus } i \text {; } \\
& f_{\text {ref }} \text { : The reference value of frequency for all } \\
& \text { generators; } \\
& V_{\text {ref }}^{i} \text { : The reference value of voltage for } \\
& \text { generator } i \text {; } \\
& m_{i}: \quad \text { Active droop coefficient at bus } i \\
& n_{i} \text { : Reactive droop coefficient at bus } i \text {; } \\
& X: \quad \text { Line Reactance; } \\
& R: \quad \text { Line Resistance; } \\
& P_{g_{i}}, Q_{g_{i}}: \quad \begin{array}{l}
\text { Active and reactive powers generated at } \\
\text { bus } i
\end{array} \\
& \delta_{i} \text { : The angle of the voltage at bus } i \text {; } \\
& N B \text { : Number of buses; } \\
& N G \text { : Number of generators in VSI mode; } \\
& P_{\text {load }_{i}}, Q_{\text {load }_{i}}: \quad \text { Active and reactive loads at bus } i \text {; } \\
& P_{\text {loss }}, Q_{\text {loss }}: \text { System's active and reactive power } \\
& \text { losses; } \\
& \text { Active and reactive powers calculated } \\
& P_{\text {calc }_{i}}, Q_{\text {calc }_{i}}: \quad \text { during the power flow convergence at bus } \\
& \Delta P_{i}, \Delta Q_{i}: \quad \text { Active and reactive power mismatches at }
\end{aligned}
$$

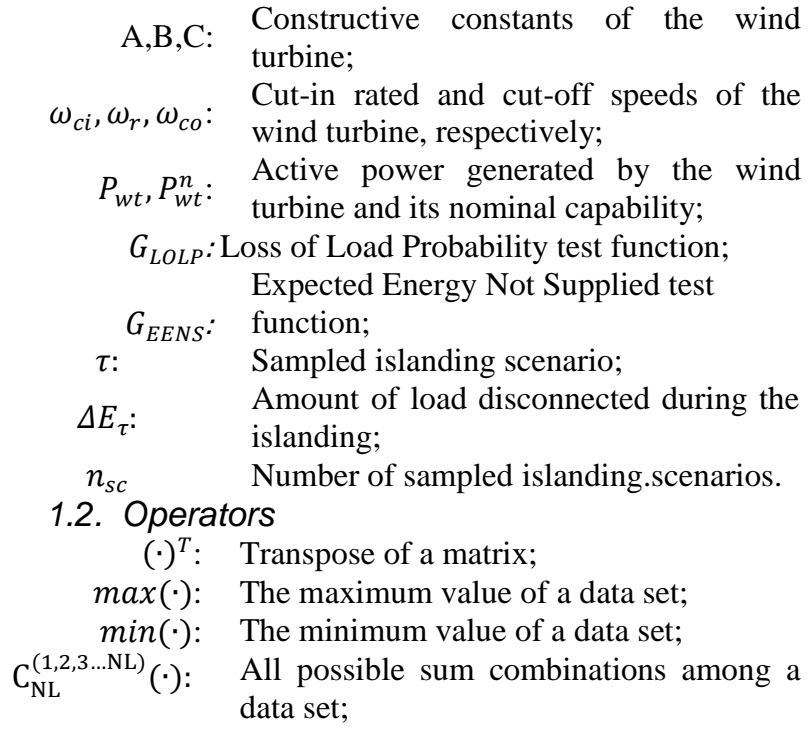

\section{Introduction}

The constant worldwide concerns regarding the increase in demand and the necessity to reduce the greenhouse gases emissions are common challenges in the evolution of electrical power systems. In this way, microgrids have become a potential solution to integrate renewable energy sources with safety and quality in the supply of electrical energy in power systems.

A microgrid is a group of interconnected loads and distributed resources, energy storage capacity and with clearly defined boundaries. These features allow a microgrid to act as a single controllable entity of the power system, being able to operate in both grid-connected and islanded modes. The high presence of renewable energy sources, telecommunication infrastructure, data processing, and autonomous operation capability are its main characteristics $[1,2]$.

Microgrids may work connected to the main grid or in the islanded mode when disconnection from the primary network drives the microgrid to supply its load according to a priority definition. A combination of secondary control, 
storage devices and load shedding strategies can be used in the islanded mode to avoid large frequency and voltage excursions. For this sake, the role of the MGCC (MicroGrid Central Controller) is to assume the full and autonomous operation of the microgrid, managing all active and passive agents at the system $[3,4]$.

In accordance with [5] and [6], the MGCC has a defined hierarchy of control composed by three levels. The first level constitutes the natural response of voltage and frequency under active and reactive power variations, commonly implemented through droop method inside the electronic interface. The second one is responsible for reestablishing voltage and frequency through the changes of set-points of generators' terminal frequency and voltage, and the tertiary level is responsible for controlling the power flow based on economic goals. Concerning these levels, only the first one is locally controlled at generators, while the others are functions implemented at the MGCC.

The possibility to have a microgrid operating only with the first level may be considered. This assumption may be especially useful in small and poor communities that lack financial resources to implement a robust telecommunication and data processing infrastructure, necessary for secondary control performance [7][8]. In these cases, if the islanded system does not have enough generation to supply its demand, load shedding may take place.

Load shedding in microgrids is treated in [9-16], and under-Frequency Load Shedding (UFLS) is approached, i.e., the UnderVoltage Load Shedding (UVLS) is not taken into account. Reference [13] proposes a Markov decision process to obtain optimal load shedding when a microgrid operates with limited resources, while [14] presents a centralized load shedding algorithm based on state estimator. In [15], a twolevel strategy load shedding based on a decentralized approach is proposed to frequency support in remote microgrids. Classes of priority of loads are considered in [16] and [17]. UFLS is essentially a global control action that equals generation and load.

UnderVoltage Load Shedding, on the other hand, may be effective as a local approach [18]. Reference [19] proposes a Jacobian matrix modification to determine the amount of active and reactive load shedding on each bus when large power systems are considered. This modification was complemented in [20], where the authors use the Tangent Vector(TV) technique to choose the buses to take UVLS in order to restore the voltage in high priority buses. However, in microgrids, this statement may not make sense, since the systems have a radial topology and the circuit breakers binary, i.e., connect or disconnect the whole load in each bus [21], which do not necessarily occur in these previous works.

Dynamic analysis of load shedding is explored in [9$11,22,23]$. Indeed, dynamic issues play a crucial role on load shedding schemes. However, static analyses have also their importance, mainly when planning studies are performed. The time response of the system is low, so, a static approach can be used to this end. Another important point is the possibility of using stochastic tools for the planning or the analysis of predictive operations.

Furthermore, [24] states the stability concept in microgrids operating in islanded mode. This reference evidences the need to consider both the amount and location of load shedding to minimize transient instability in any operational decision. Besides, advanced methods to monitor data and network model play a crucial role in this approach.

Some services like telecommunications, transportation, and health care must be uninterruptible, i.e., the energy supply must be ensured even in an adverse scenario [25]. In this sense, the possibility to use load shedding strategies, considering the restriction of the continuous energy supply to high priority loads is a motivation of this paper.

The operating point of a microgrid along time may be adequately determined by employing a proper load flow at each point. In microgrids context, there are some differences from a conventional system that must be considered [26-29], especially the load's and renewable power generation's intermittency.

The acceptable ranges of voltage and frequency for islanded active distribution systems are related in [30] and adopted here. Values outside these ranges trigger the proposed load shedding scheme.

Thus, the frequency and the voltages for all buses are controlled by the minimum load shedding in lower priority loads, maintaining these values in a safe range and guarantying the energy supply to higher priority loads. The focus of this work is the steady-state analysis from load flow and, therefore, dynamic aspects are not considered.

In the exposed context, the main contributions of this paper are highlighted:

- A control scheme with load shedding capacity to preserve the high priority loads of the system in any islanding scenario;

- A complementary load shedding hierarchical policy for voltage and frequency by using the UVLS (based on the Tangent Vector technique) and UFLS (based on the nominal capacity and droop characteristics of generators) approaches;

- A comparison between the UVLS with and without the use of Tangent Vector technique, highlighting its dependence coordinated with loads priorities;

- A validation approach based on the Monte Carlo Simulation (MCS) [21]. This last contribution reveals the adequate performance of the proposal for different load and generation scenarios and its superiority to a state-ofthe-art load shedding solution that does not consider the priority of loads.

The remainder of the paper is organized as follows. Section III brings the proper microgrid load flow implemented within a time domain. Section IV shows the proposed strategies based on load shedding with priority loads to correct microgrid's frequency and voltage. Additionally, the Monte Carlo Simulation is also performed to validate these strategies of load shedding. The results are presented in Section V, considering a random day separately to show the load shedding strategies and a Monte Carlo simulation to prove their accuracy. A comparison with the results of he control strategy proposed in [19] that does not consider priority loads and TV technique is also incl lete 1 , revealing the superior performance of the proposal methodology allied with the usage of TV technique to set lower priority buses to load shedding. Finally, Section VI exposes the main conclusions of the work. 


\section{Microgrids Operation in Islanded Mode}

An essential element for a microgrid survival is the MGCC, generally located at the primary substation. A policy of management resources and agents must ensure the power supply following regulated limits as long as possible. Also, the power flow algorithm is an essential tool in the state variables determination in any part of the system.

In the system's topology issues, it is not usually the direct connection of generators into the microgrids but connected through Power Electronic Converters (PEC). In microgrids, the converters may commonly work in two modes of operation:

- Current Source Inverter (CSI) mode: PECs in CSI mode operates as grid-following converters, i.e., they inject a defined amount of power. This can be, for instance, the maximum power extracted from a renewable energy source.

- Voltage Source Inverter (VSI) mode: PECs in VSI mode operates as grid-forming converters by controlling the voltage and frequency at their output side. This is typically done by using the droop method, which modifies the frequency and voltage according to the supplied active and reactive power, respectively.

In the droop method, the frequency and voltage outputs of the inverter follow the power demand linearly. These outputs are modeled as $[3,26,31]$ :

$$
\begin{gathered}
f=f_{\text {ref }}-m_{i} P_{g_{i}} \\
V_{i}=V_{\text {ref }}^{i}-n_{i} Q_{g_{i}}
\end{gathered}
$$

The droop method does not require the use of communication at the first level. Fig. 1 shows the droop method operating according to the power demand of a generic system. Additionally, the islanded operation points before and after the load shedding are also included.

There are two kinds of non-dispatchable active power generation sources considered in this paper: solar and wind. The static models of photovoltaic panels and wind turbine are described in [32] and [33], respectively. The available power from each photovoltaic panel is a function of solar irradiance and the bus voltage. As for a wind turbine, its available power is a function of wind speed. Both are considered as negative loads in the load flow formulation. Fig 2(a) and Fig.2(b) respectively depict the static models of photovoltaic panels (PV) and wind turbines (WT), considered in this work.

To solve the load flow, the Newton-Raphson Method (NRM) was used by incorporating (1)-(2) in the convergence process. The step-by-step of the time-continuous load flow complemented by the droop equations is based on [29]. The Levenberg-Marquardt Method [34] to improve the convergence, engaged by the $\mathrm{R} / \mathrm{X}$ ratio, and non-dispatchable sources as negative loads are added to characterized a microgrid, as described below.

\subsection{Islanded Microgrids Load Flow}

Power flow algorithms are widely used in planning and operation analysis. In microgrids, thanks to the data processing and telecommunication infrastructure, it can be implemented inside the MGCC to determine the state variables in any part of the grid. The NRM is used for transmission systems and may be used for distribution ones.

Unlike the transmission system, the load flow in islanded microgrids has some peculiarities. As addressed in
[26-29], the main problems associated with the formulation of load flow in islanded microgrids by conventional methods are:

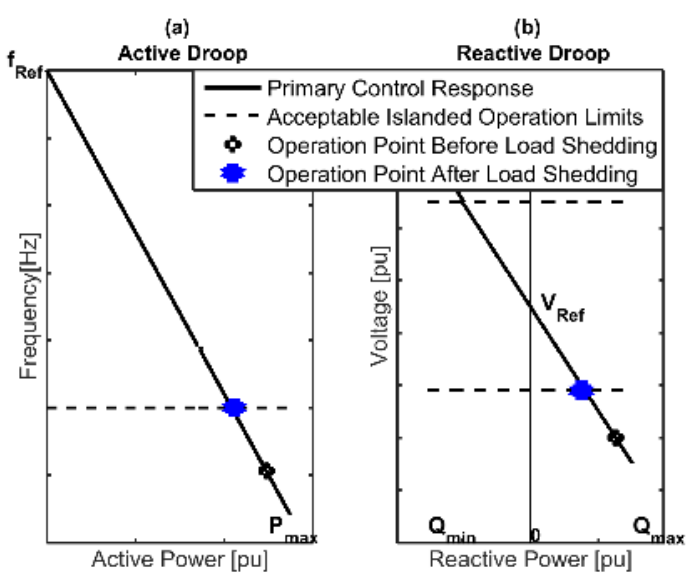

Fig. 1. The droop method: (a) frequency range and (b) voltage range by active and reactive powers, respectively.
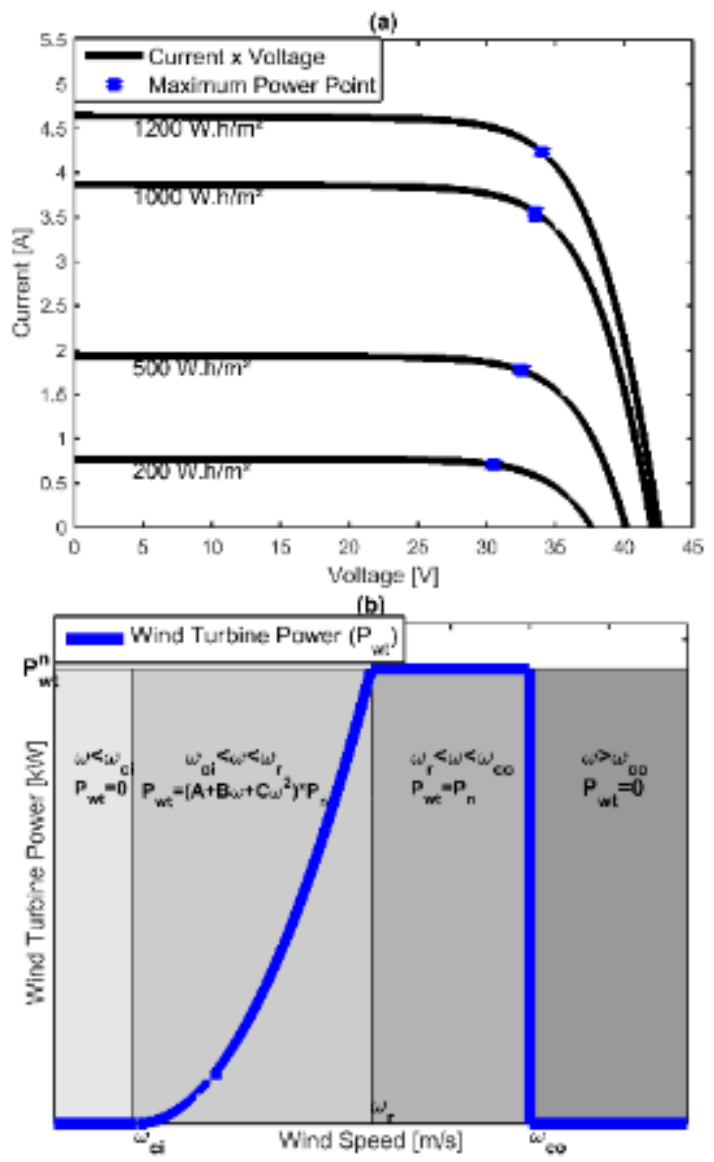

Fig. 2. (a) Photovoltaic Panel static model, (b) Wind Turbine static model.

- There is no swing bus;

- The terminal voltages at the sources may vary;

- The frequency is not kept constant;

- The X/R ratio can be small, which hinders the convergence using conventional methods to solve the load flow;

- The loads tend to vary in accordance to the frequency and voltage. Here, the load model proposed in [29] is used. 
This paper presents a time-continuous load flow based on the methodology proposed in [29]. Briefly, the load flow problem in microgrids is a function of all voltage magnitudes, inclusive the generators, the frequency, and the angles, as exposed in (3).

$$
f\left(\boldsymbol{\delta}, \boldsymbol{V}, V_{1}, f\right)=0
$$

where $\delta$ are the angles and $V$ are the voltages magnitudes of all buses, except the Bus 1, that has the voltage's magnitude represented by $V_{1}$ and is the only one with the defined angle. $f$ is the frequency of the system.

Two approaches form the set of equations. Equations (4)-(5) apply to all buses, whereas equations (6)-(7) regard the generation/load net balance.

$$
\begin{gathered}
\Delta P_{k}=\left(P_{g_{k}}-P_{\text {load }_{k}}\right)-P_{\text {calc }_{k}} ; k=(1,2,3 \ldots N B) \\
\Delta Q_{k}=\left(Q_{g_{k}}-Q_{\text {load }_{k}}\right)-Q_{\text {calc }_{k}} ; k=(1,2,3 \ldots N B) \\
\Delta Q_{\text {sys }}=\sum_{k=1}^{N B} Q_{\text {load }_{k}}+Q_{\text {loss }}-\sum_{l=1}^{N G} Q_{g_{l}} \\
\Delta P_{\text {sys }}=\sum_{k=1}^{N B} P_{\text {load }_{k}}+P_{\text {loss }}-\sum_{l=1}^{N G} P_{g_{l}}
\end{gathered}
$$

According to the proposed methodology, the generation from grid-forming converters tends to sources tends to vary as a function of the voltage and frequency. These values can be obtained by rewriting (1) and (2), which results in:

$$
\begin{gathered}
P_{g_{k}}=\frac{f_{r e f}-f}{m_{k}} \\
Q_{g_{k}}=\frac{V_{r e f}^{k}-V^{k}}{n_{k}}
\end{gathered}
$$

To update the variables during the convergence process, equations (4)-(7) are linearized concerning the state variables, which results in the Jacobian matrix exposed below. The NRM to microgrid is performed by equation (10) [29].

$$
\begin{aligned}
& \begin{array}{llll}
\frac{d \boldsymbol{P}}{d \boldsymbol{\delta}} & \frac{d \boldsymbol{P}}{d \boldsymbol{V}} & \frac{d \boldsymbol{P}}{d V_{1}} & \frac{d \boldsymbol{P}}{d f}
\end{array} \\
& \left.\left[\begin{array}{c}
\Delta \boldsymbol{\delta} \\
\Delta \boldsymbol{V} \\
\Delta V_{1} \\
\Delta f
\end{array}\right]=\begin{array}{ccccc}
\frac{d \boldsymbol{Q}}{d \boldsymbol{\delta}} & \frac{d \boldsymbol{Q}}{d \boldsymbol{V}} & \frac{d \boldsymbol{Q}}{d V_{1}} & \frac{d \boldsymbol{Q}}{d f} & \Delta \boldsymbol{P} \\
\frac{d Q_{s y s}}{d \boldsymbol{\delta}} & \frac{d Q_{s y s}}{d \boldsymbol{V}} & \frac{d Q_{s y s}}{d V_{1}} & \frac{d Q_{s y s}}{d f} & \Delta Q_{s y s} \\
\left.\Delta P_{s y s}\right\rfloor
\end{array}\right] \\
& \left\lfloor\begin{array}{llll}
\frac{d P_{s y s}}{d \boldsymbol{\delta}} & \frac{d P_{s y s}}{d \boldsymbol{V}} & \frac{d P_{s y s}}{d V_{1}} & \left.\frac{d P_{s y s}}{d f}\right\rfloor
\end{array}\right.
\end{aligned}
$$

However, in microgrids, the resistance of the line is predominant, i.e., the $\mathrm{X} / \mathrm{R}$ ratio is low, making the couplings $\mathrm{P} / \mathrm{f}$ and $\mathrm{Q} / \mathrm{V}$ no longer guaranteed. Therefore, traditional power flow methods may fail. The Levenberg-Marquardt Method [34] is employed to improve the convergence process, mitigating this problem.

Additionally, due to the Jacobian matrix sparsity and the lack of buses with a fixed voltage, this matrix becomes badly conditioned. To overcome this problem, the update of the state variable is done by Levenberg-Marquardt Method, as:

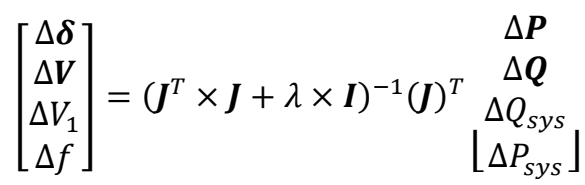

This method efficiently avoids convergence problems. The convergence of NRM results in the state of the microgrid for a specific operating scenario and the performance of primary control, i.e., the droop response of the generators.
The increase of load results in frequency and voltage reduction, as shown in Fig. 1, which may result in an infeasible operating condition from the technical and regulatory point of view. Thus, the voltage level and the frequency must be recovered to fair values mainly in high priority consumers. For this sake, a scheme of load shedding to save high priority loads proposed in this work is presented in the next section.

The solar incidence, wind speed, and the loads' profile are taken into account as entries of the problem, modifying the scenario of load and generation during the islanding mode. So, for each instant, the load flow for microgrid is solved.

Table 1. Classes of Loads Priorities.

\begin{tabular}{ccc}
\hline Class & Priority & Services \\
\hline & & Substations, Hospitals, \\
1 & High & Telecommunication and \\
& & Transportation Services \\
2 & Medium & Commercial \\
3 & Low & Residential \\
\hline
\end{tabular}

\section{The Load Shedding Scheme}

After the load flow convergence, the frequency and voltage are verified. If necessary, load shedding is employed to recover the voltage and the frequency within limits. The proposed load shedding scheme considers a hierarchical order based on the priority of each class of load to restore the operating point.

According to [17], the loads can be divided into three different classes, related with the degrees of priority(high, medium and low), as shown in Table 1. This classification is used in this paper to sort the load shedding in microgrids to keep the energy supply uninterruptable to high priority consumers.

The load shedding scheme presented in this paper has three fundamental premises:

- The load shedding comprises simultaneous both active and reactive power in each bus. This is done by considering the coupling between these values in a load;

- The load shed in a bus is made as a whole. Thus, it is not feasible the partial load shedding, that is, the circuitbreakers act in a binary way.

- The decision of load shedding is performed before the actuation of voltage and frequency protective devices.

\subsection{UnderVoltage Load Shedding (UVLS)}

Differently from the frequency, voltage is a local variable. This implies that the UVLS must be executed locally. A sensitivity analysis was adopted to accomplish this goal.

Generally, the load shedding by undervoltage is done on the same bus that presents low values of voltage [19]. This may change if this bus is of a high priority one. In this case, considered in this paper, more buses may take load shedding to mitigate the problem.

The Tangent Vector (TV) is used in the literature as the predictor step of the continuation method [35-37], used to calculate the load margin of a system. It may also be used to determine the critical bus of the system. The critical bus is the one most sensitive in the system concerning a load variation. Thus, it identifies the buses that can improve the voltage through a load shedding. This approach is used here 
to help with the identification of the buses most likely to load shedding. The load's priorities are taken into account separately.

It is worthy, however, to mention that the most sensitive bus observed here is a consequence of the system's topology and the operation point. Further, this statement does not necessary means that this bus presents the lowest voltage level [20].

The TV is given by (12):

$$
[T V]_{i}=\left[\begin{array}{cc}
\boldsymbol{H}^{\prime} & \boldsymbol{N}^{\prime} \\
\boldsymbol{M}^{\prime} & \boldsymbol{L}^{\prime}
\end{array}\right]^{-1} \begin{gathered}
0 \\
P_{\text {load }_{i}} \\
0 \\
\\
\end{gathered}
$$

in (12), $H^{\prime}, M^{\prime}, N^{\prime}$ and $L^{\prime}$ are the submatrices from the Jacobian matrix when it is reduced in the same way presented in [38]. Hence, only components of active and reactive power in relation to voltages and angles are explored.

The values of the Tangent Vector of all buses are sorted in descending order, separately for each class of priority. Then, it is possible to determine a hierarchy composed of the most sensitive buses, based on the voltage profile of the system and the classes of priority.

Therefore, the amount of load the must be shed by undervoltage is applied initially to class 3 in accordance with Tangent Vector magnitude of buses. If this action is not enough, it is applied to class 2, and finally, if still necessary, to class 1 , as depicted in (13).

$$
\Delta \text { Load }_{\text {volt }}=\left\{\begin{array}{c}
(P, Q)_{[\max (V T) \ldots \min (V T)]} ; \text { prior }=\text { Low } \\
(P, Q)_{[\max (V T) \ldots \min (V T)]} \text { prior }=\text { Medium } \\
(P, Q)_{[\max (V T) \ldots \min (V T)]} ; \text { prior }=\text { High }
\end{array}\right.
$$

The load's priority-based load shedding may change the critical bus of the system. For this reason, the TV must be updated in each time sample. The computational demand is low, because the Jacobian matrix has already been implemented in the load flow, so this calculation is not a drawback.

\subsection{UnderFrequency Load Shedding (UFLS)}

If the frequency is still in infeasible values after the performance of UVLS, the UFLS is performed.

For each dispatchable unit, the generation in the limit frequency can be rewritten from (8), having the frequency $(f)$ defined in the limit. This is done like exposed in (14).

$$
P g_{\text {lim }_{i}}=\frac{\left(f_{\text {ref }}-f_{\text {lim }}\right)}{m_{i}}
$$

where $f_{\text {lim }}$ is the adopted limit frequency, and $P g_{\text {lim }}$ is the maximum dispatchable power at generator $i$ to maintain the frequency of the system at the limit.

Hence, the amount of load necessary to be shed to return the frequency within the limit can be defined by the difference between the total amount of loads and losses, and the entire available generation at the frequency limit, which is expressed in (15).

$$
\Delta_{\text {Load }_{\text {freq }}}=\left(\sum_{i=1}^{N B} P_{\text {load }_{i}}+P_{\text {loss }}\right)-\sum_{i=1}^{N G} P_{\text {lim }_{i}}
$$

UFLS can be executed in any part of the system since the frequency is a global variable. So, to distribute $\Delta$ Load $_{\text {freq }}$ among the buses, a sum combination scheme is employed for each priority class of bus, as shown in (16).

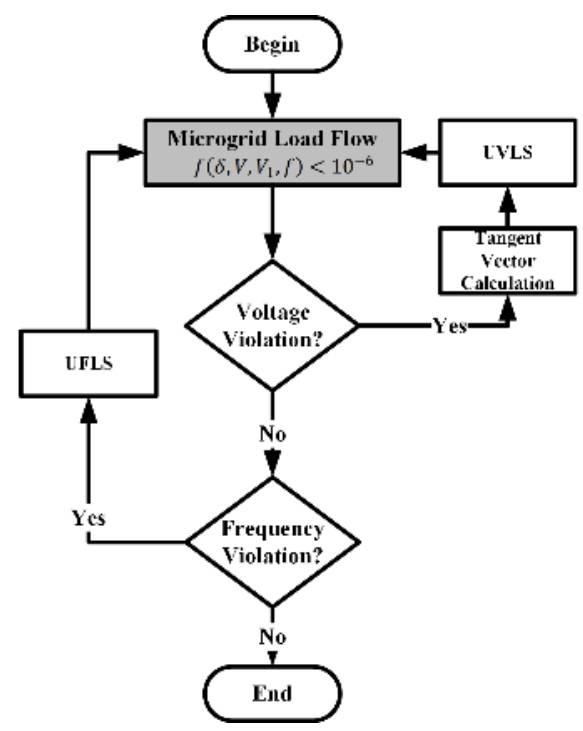

Fig. 3. Flowchart of the proposed load shedding scheme.

$$
\begin{aligned}
\Delta \text { Load }_{\text {freq }} \leq \min & \sum_{i=1}^{n \in C_{N L}^{(1,2,3 \ldots N L)}} P_{\text {load }_{i} \text { prior }} \\
\text { prior } & \in(\text { Low }, \text { Medium, High })
\end{aligned}
$$

In the UFLS, the hierarchy formed by low, medium and high priorities of loads is respected. Then, $\Delta$ Load $_{\text {freq }}$ is firstly divided among low priority buses. If it is not enough to restore the frequency value, load shedding is performed at the medium priority loads. Finally, if it is still out of the nominal range, the lowest high priority loads are considered for load shedding.

Fig. 3 shows the flowchart that summarizes the proposed approach of load shedding, including the UVLS and UFLS corrective actions. Note that the algorithm ends when both voltage and frequency are within limits.

\subsection{Monte Carlo Simulation}

Since the wind and solar resources, as well as the loads, vary with time, the instant and the duration of the disconnection become fundamental for determination of the load shedding. Thus, the Monte Carlo Simulation is applied in order to quantify the performance of the methodology, when a microgrid operates in islanded mode.

Therefore, this paper considers the operation of a microgrid in islanded mode in different scenarios, modelled by two random variables sequentially sampled:

- The instant of disconnection, corresponding to the moment that the microgrid changes its operation mode from grid-connected to island. Uniform distribution samples this instant during the corresponding month;

- The duration of disconnection, i.e., the time that the microgrid operates autonomously. This variable is sampled in a normal distribution.

Summarizing, the MCS consists of the sequential sampling of the initial time and duration of disconnection to create different scenarios of islanding during a considered period, a specific month for example. In these scenarios, the solar irradiance, wind speed, and loads profiles became inputs of the problem. Based on this, the time-continuous load flow analysis is performed, and the test-functions of the LOLP (Loss Of Load Probability) and EENS (Expected Energy Not Supplied) indices are calculated, as shown in Fig. 4. 


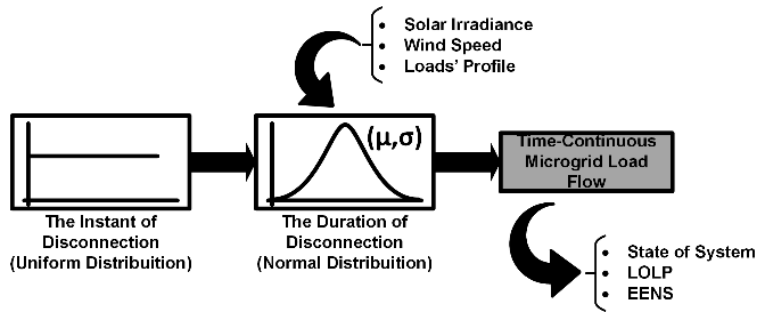

Fig. 4. Monte Carlo Simulation approach.

The test-functions for LOLP and EENS indices are implemented as exposed in (17) and (18), respectively.

$$
\begin{aligned}
& G_{\text {LOLP }}(\tau)=\left\{\begin{array}{l}
0, \text { if there is no load shedding } \\
1, \text { if load shedding is executed }
\end{array}\right. \\
& G_{\text {EENS }}(\tau)=\left\{\begin{array}{l}
0, \text { if there is no load shedding } \\
\Delta E_{\tau}, \text { if load shedding is executed }
\end{array}\right.
\end{aligned}
$$

Both of these test functions are calculated separately to UVLS and UFLS, as well as to each class of priority group of loads. Then, LOLP and EENS indices are calculated as the expected values of the corresponding test-functions, i.e., $G_{L O L P}(\tau)$ and $G_{E E N S}(\tau)$, evaluated for each sampled scenario of islanding. Mathematically:

$$
\begin{aligned}
& L O L P=\frac{1}{n_{s c}} \sum_{\tau=1}^{n_{s c}} G_{L O L P}(\tau) \\
& E E N S=\frac{1}{n_{s c}} \sum_{\tau=1}^{n_{s c}} G_{E E N S}(\tau)
\end{aligned}
$$

This process is repeated until convergence is achieved, as exposed in [39]. For each priority class, these two indices are responsible for analyzing the system performance in islanded mode. LOLP gives the probability of occurrence of load shedding, while EENS is the expected amount of energy not supplied during the autonomous operation of the microgrid in the corresponding month.

\section{Results and Discussions}

As a way to validate the load shedding scheme proposed in this paper, the IEEE 37 Node Test Feeder [40] was used (Fig. 5). This system has an unbalanced topology, so some modifications have been made to turn it equivalent to a balanced one [41]. These new systems considerations are listed below:

- $\quad$ Only the positive sequence of the lines is considered;

- The spot loads are regarded as the average of three phases;

- $\quad$ The distributed loads along the feeder are seen as spot loads between the buses of the feeder.

- The p.u. values are calculated in the single-phase nominal base of the system, i.e., 2500/3 kVA.

This system is a passive distribution system, so, to adapt it to a microgrid, the topology of the network was complemented as following:

I. Buses 701, 724, 729, 736 and 741 have dispatchable microturbines with $m=0.0015 \frac{\mathrm{Hz}}{\mathrm{kW}}, n=0.0005 \frac{\mathrm{kV}}{V A r}$ and 0.6 p.u. of nominal power.

II. There are five wind turbines with 0.25 p.u. of nominal power at Buses 742, 731, 725, 775 .

III. There are five photovoltaic arrays at bus Buses 722 , $730,728,737,732$ with approximately 0.20 p.u. of nominal power each one.

The loads are distributed as described in Table 2. In this work, the Brazilian frequency is assumed as the reference value, i.e., $60 \mathrm{~Hz}$, with $0.5 \mathrm{~Hz}$ of tolerance to the limit. The voltage level remains in 1 p.u. as the reference value for all generators with 0.05 p.u. of tolerance as the system's voltage limit. Furthermore, the time series of renewable generation and loads profiles are obtained from a local Brazilian utility grid data base, located in Itajubá-Minas Gerais.

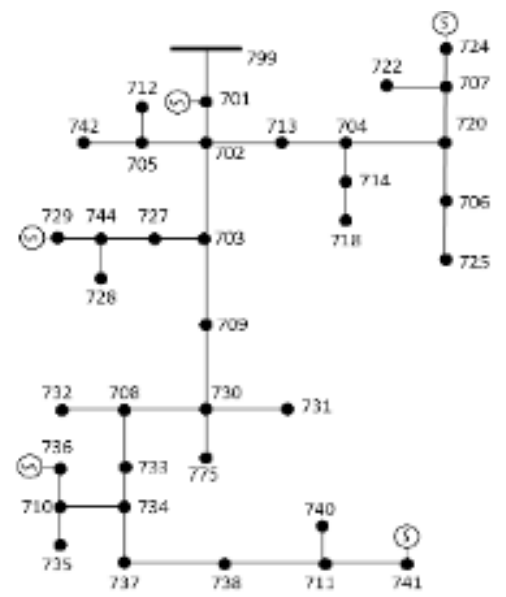

Fig. 5. The modified IEEE 37 Node Test Feeder.

Table 2. Classes of Loads' Priorities.

\begin{tabular}{cc}
\hline Priority & Bus \\
\hline & $701,703,704,705,706,707,709,711,713$, \\
Low & $718,727,728,729,732,734,735,736,738$, \\
& $741,775,799$ \\
Medium & $702,708,710,712,714,720,730,731,740,744$ \\
High & $722,724,725,733,737,742$ \\
\hline
\end{tabular}
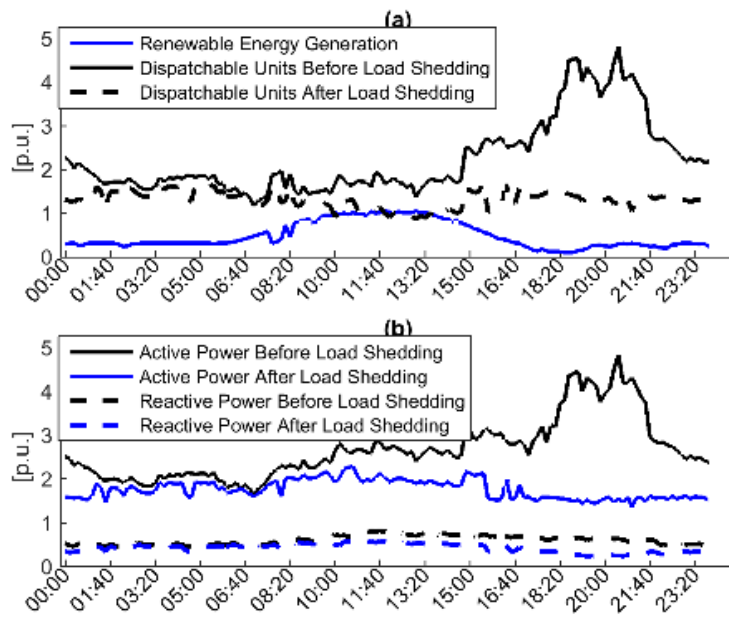

Fig. 6. (a)Generation, and (b) load profiles of the system before and after the load shedding.

As a way to better illustrate the results, it has been divided into two parts. The first one presents performance tests in a defined time-based domain, while the second shows the Monte Carlo Simulation analysis, giving an estimative about the probability and the amount of load shedding. All results are stated in the base case, after UVLS and UFLS conditions.

\subsection{Time Continuous Load Flow Analysis}

The time-continuous load flow analysis is performed in a 24 hours simulation scenario, and it considers steps of 10 minutes. This is done to show the response of load-shedding strategies. Fig. 6 shows the load and generation curves, the 
result of the execution of the algorithm during the $24 \mathrm{~h}$ timebased. It is possible to note that during the whole simulation the generation performance tends to follow the load characteristic, being slightly higher due to the losses. Moreover, due to an increase in renewable power generation, power dispatches are reduced.

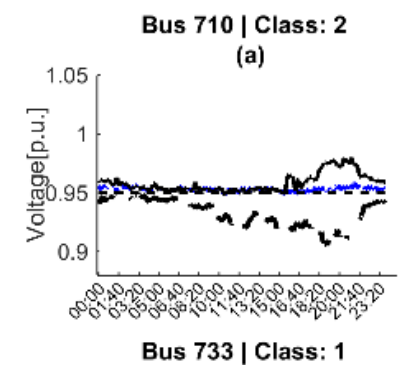

(c)

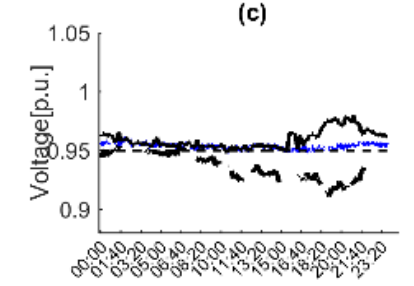

Fig. 7. The lowest voltage profile of the system.

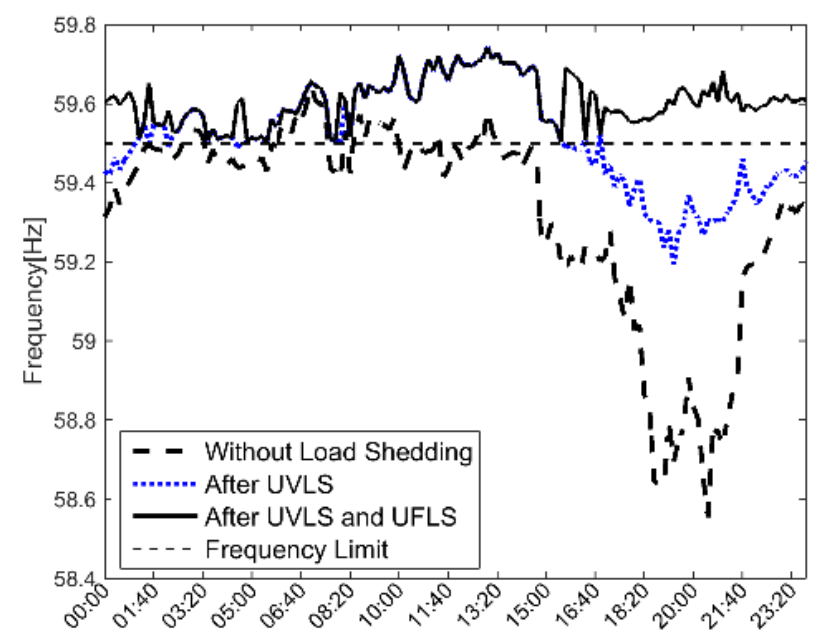

Fig. 8. The frequency profile of the system.

The voltages are monitored for each time step after the load convergence. If any bus presents a low voltage, the UVLS is employed. Buses 710, 733, 734 and 737 presented the lowest values during the considered day, which are illustrated in Figs. 7(a)-(d). Since some of them are medium and high priority loads, the load shedding must not be executed locally - except at Bus 734. The Tangent Vector was used in the determination of the most susceptible buses that can take load shedding to preserve the load of these medium and high priorities buses. Hence, the priority of buses in the load shedding process is respected, so the hierarchy defined by (12) is applied.

There is a small and unnecessary increase in the voltages after the UVLS performance between 16:00 and 23:50. This occurs due to the frequency is still under the limit, which is depicted in Fig. 8. Such a figure describes the sequence (base case, UVLS, UFLS), so one can see that the UFLS restores the frequency and improves the voltage. If necessary, even after UVLS performance, the UFLS takes place. Fig. 8 shows the frequency profile of the system after only UVLS and after UVLS and UFLS performances. It is possible to note that the frequency is within its limit after the UFLS is applied. This is done to preserve both the frequency and the voltage employing a minimum load shedding. Note also in Fig. 8 that the low frequency value observed could be a problem, tripping some wind generation units. However, because both UVLS and UFLS are considered, this low frequency is not observed. The actual behavior observed is the one associated with the bold solid line, when this frequency violation does not take place.
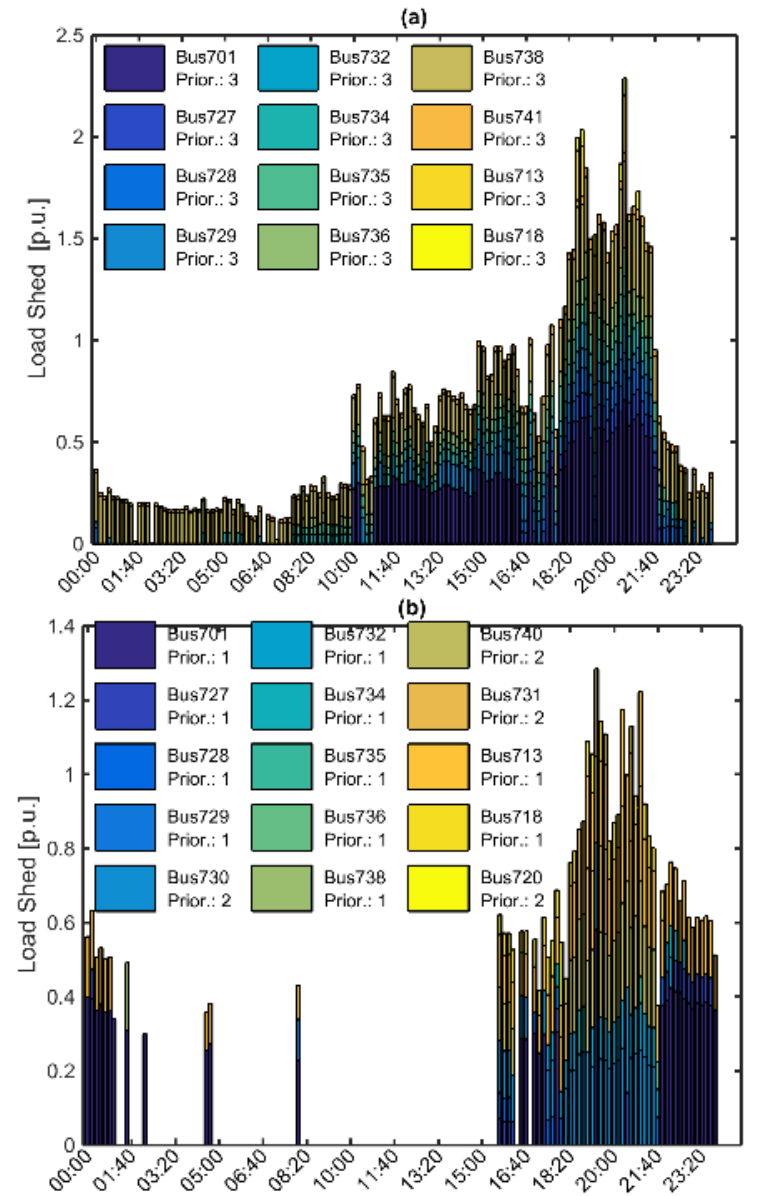

Fig. 9. (a) Buses under UVLS with TV and, (b) Buses that experienced UFLS.

The distributions of load shedding by undervoltage and underfrequency are shown in Fig. 9(a) and Fig.9(b), respectively. From Fig. 8(a), the load shedding of low priority loads is responsible for restoring the voltage profile of medium and high priority consumer within acceptable limits. Moreover, this action improves the frequency, as shown in Fig. 8. On UFLS, when there is a heavy load scenario (lower frequency values), it is required to shed medium priority loads, as shown in Fig.9(b). This occurs due to the massive disconnection of low priority consumers during the UVLS verification. The medium priority load shedding is the main responsible for frequency correction.

Fig. 10 shows the performance of UVLS without the use of the Tangent Vector. The undervoltage problem is fixed. However, some consumers with certain priority degree are disconnected. This methodology was proposed in [19], where the undervoltage problem is treated only in a local way. In this sense, the results displayed in Fig. 9(a) render the Tangent Vector-proposed methodology as useful since the undervoltage is overcome by preserving the high priority loads. It is important to notice that, even though Buses 733, 
737 and 710 present the lowest voltage level, they suffer no load shedding, because of their high priority. In this case, the Tangent Vector technique was applied to determine a region of buses capable of correcting the voltage.

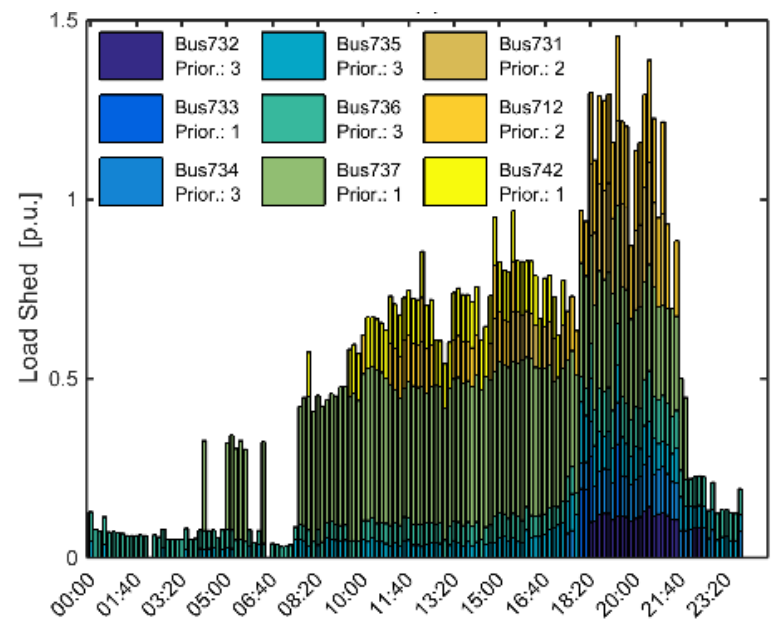

Fig. 10. Buses under UVLS without TV technique.

One could argue that overvoltage and overfrequency should be addressed. These are possibilities that may take place when the system presents a high penetration of renewable energy sources. The scenarios considered here do not allow this operating condition. Because the penetration of renewable sources is smaller than load, overfrequency and overvoltage are not an issue. However, a high level of renewables penetration could create overvoltage and overfrequency problems. This may be faced as a hosting capacity problem [42], and storage devices could be an option. On the other hand, if no storage is considered, as in this paper, the proposed methodology would handle the problem according to Section 4, where the strategy to approach both problems is detailed.

\subsection{Monte Carlo Simulation Analysis}

Finally, to assess the efficiency of the load shedding strategy proposed here, the Monte Carlo Simulation is used. The LOLP and EENS indices are monitored until the convergence of the simulation. Through these indices, it is possible to determine the load shedding (if any) of each priority class considered separately. Besides, these indices provide an estimate about how much load has been shed, to maintain the operations scenarios within standards defined by regulatory agencies during the island.

In Tables 3 and 4, the values of LOLP and EENS regarding UVLS and UFLS are exposed for a month. Further, a comparison between the approaches with (proposed methodology) and without the TV technique (proposed in [19]) is also exposed. The MCS has been executed with uniform probability to the islanding instant, and a normal distribution probability to its duration. The values of average and standard deviations are 50 and $20 \mathrm{~min}$, respectively.

Table 3 shows that the probability of load shedding in high priority consumers is zero when the TV technique is considered. This implies in no load shedding in this kind of consumers. Moreover, the total of EENS with TV is higher than without one, however, in this case, the high priority consumers experience load shedding to correct the system's voltage profile. This evidences the necessity to consider a sensitivity analysis to overcome the undervoltage problem without load shedding in special consumers.

Table 3. LOLP and EENS to UVLS

\begin{tabular}{|c|c|c|c|}
\hline & With TV & $\begin{array}{c}\text { Without TV } \\
{[19]} \\
\end{array}$ & $\Delta[\%]$ \\
\hline & \multicolumn{3}{|c|}{ LOLP } \\
\hline Low & 1 & 0.6 & $-40 \%$ \\
\hline Medium & 0.15 & 0.47 & $+68 \%$ \\
\hline \multirow[t]{2}{*}{ High } & 0 & 0.34 & --- \\
\hline & \multicolumn{3}{|c|}{ EENS $[M W h]$} \\
\hline Low & 1.9 & 0.53 & $-72 \%$ \\
\hline Medium & 0.54 & 0.63 & $+16 \%$ \\
\hline High & 0 & 0.15 & ---- \\
\hline
\end{tabular}

Table 4. LOLP and EENS to UFLS

\begin{tabular}{cccc}
\cline { 2 - 4 } & With TV & $\begin{array}{c}\text { Without TV } \\
{[19]}\end{array}$ & $\Delta[\%]$ \\
\cline { 2 - 4 } & \multicolumn{3}{c}{ LOLP } \\
Low & 0.67 & 0.35 & $47 \%$ \\
Medium & 0.51 & 0.47 & $-8 \%$ \\
High & 0 & 0.07 & ---- \\
\cline { 2 - 4 } & \multicolumn{3}{c}{ EENS[MWh] } \\
Low & 0.38 & 0.58 & $+34 \%$ \\
Medium & 0.68 & 0.35 & $+48 \%$ \\
High & 0 & 0.49 & ----
\end{tabular}

In Table 4, the EENS with TV technique presents the values to medium priority loads more significant than the corresponding of low priority ones in Table 3 . This is because most of the lower priority load has been shed to correct the voltage. Therefore, the frequency correction becomes a responsibility of load shedding in medium priority loads.

\section{Conclusions}

This paper proposed a methodology to guarantee the safe and quality energy supplying of high priority loads in islanded microgrids. Based on undervoltage and underfrequency, a hierarchical load shedding was proposed to maintain the frequency and the voltage in all buses in acceptable values without secondary control.

The main contributions of the paper are pointed out below:

- To determine the state of the microgrid, a timecontinuous load flow based on Newton-Raphson Method was implemented. This algorithm was adapted from the literature to handle the characteristics of convergence in distribution systems by Levenberg-Marquardt Method. No convergence problems were observed in any scenario.

- Two different load shedding strategies were carried out to restore the safe operation of the microgrids. First, load shedding for voltage deviation was addressed in a sensitivity analysis. Then, a load shedding scheme for under-frequency was proposed and tested. Hence, the microgrid can operate in an emergency, preserving the operation limits by load shedding schemes.

- Associated with the performance of load shedding schemes, a Monte Carlo Simulation was used to give an estimative of the load shedding. The indices 
LOLP and EENS were monitored. These indices can, for example, help the operator in predictive action during the islanding;

- Finally, the results show the efficiency of the proposed method compared to a traditional methodology of UVLS that consider a Jacobian matrix modification. The voltage and frequency are restored to a safe range, and the energy was supplied to the high priority loads uninterruptedly. It is worthy to mention that the load shedding was made as a whole at the bus, differently from large systems, where the load shedding can be made partially at the substation.

Some open questions that can be studied in future works are listed below:

- The possibility of islanded operation in active distribution systems with lack of resources;

- A coordination between load shedding and secondary control;

- Predictive actions based on stochastic analysis;

- Social aspects in load supply, mainly in poor and small communities, and others.

\section{Acknowledgments}

This work was supported in part by $\mathrm{CNPq}$ [grant number 142026/2016-6], CAPES [grant number 1575647], FAPEMIG [grant number PPM-00685-17] and INERGE.

\section{References}

[1] Zambroni de Souza, A.C., Oliveira, D.Q., Marujo, D., Portelinha Jr., F.M., de Nadai N., B.: 'Overview on Microgrids: Technologies, Control and Communications', in Azzopardi, B. (Ed.): 'Sustainable Development in Energy Systems' (Springer, 2017, 1st edn.), pp. 1-18

[2] Parhizi, S., Lotfi, H., Khodaei, A., Bahramirad, S.: 'State of the Art in Research on Microgrids: A Review',IEEE Access, 2015, 3, pp. 890-925.

[3] Peças Lopes, J.A., Moreira, C.L., Madureira, A.G.: 'Defining Control Strategies for MicroGrids Islanded Operation',IEEE Trans. Power Syst., 2006, 21, (2), pp. 916-924.

[4] Zambroni de Souza, A.C., de Nadai N., B., Portelinha Jr., F.M., Oliveira, D.Q., Marujo, D.: 'Microgrids Operation in Islanded Mode', in Azzopardi, B. (Ed.): 'Sustainable Development in Energy

Systems' (Springer, 2017, 1 st), pp. 193-215

[5] Guerrero, J.M., Vasquez, J.C., Matas, J., García de Vicuña, L., Castilla, M.: 'Hierarchical Control of Droop-Controlled AC and DC Microgrids - A General Approach Toward Standardization',IEEE Trans. Ind. Electron., 2011, 58, (1), pp. 158-172.

[6] IEEE: 'IEEE Standard for the Specification of Microgrid Controllers', (2017)

[7] Souza, M.F.Z. de: 'On Rural Microgrids Design - A Case Study in Brazil', in 'IEEE PES Innovative Smart Grid Technologies Latin America' (2015), pp. 160-164

[8] Shahidehpour, M., Pullins, S.: 'Microgrids, Modernization and Rural Electrification',IEEE Electrif. Mag., 2016, (1), pp. 2-6.

[9] Hajimohammadi, N., Bevrani, H.: 'Load Shedding in
Microgrids', in '18th Electric Power Distribution Conference' (2013), pp. 1-7

[10] Hong, Y., Hsiao, M., Chang, Y., Lee, Y., Huang, H.: 'Multiscenario Underfrequency Load Shedding in a Microgrid Consisting of Intermittent Renewables',IEEE Trans. Power Deliv., 2013, 28, (3), pp. 1610-1617.

[11] Issicaba, D., Augusto, M., Resende, F.O., Santos, B., Lopes, J.A.P.: 'Long-Term Impact Evaluation of Advanced Under Frequency Load Shedding Schemes on Distribution Systems with DG Islanded Operation',IEEE Trans. Smart Grids, 2019, 10, (1), pp. 238-247.

[12] Zhou, Q., Li, Z., Wu, Q., Shahidehpour, M.: 'TwoStage Load Shedding for Secondary Control in Hierarchical Operation of Islanded Microgrids', IEEE Trans. Smart Grid, 2018, 3053, (c), pp. 1-8.

[13] Gao, H., Chen, Y., Xu, Y., Liu, C.: 'Dynamic load shedding for an islanded microgrid with limited generation resources',IET Gener. Transm. Distrib., 2016, 10, (12), pp. 2953-2961.

[14] Karimi, M., Wall, P., Mokhlis, H., Terzija, V.: ‘A New Centralized Adaptive Under - Frequency Load Shedding Controller for Microgrids based on a Distribution State Estimator',IEEE Trans. Power Deliv., 2017, 32, (1), pp. 370-380.

[15] Pashajavid, E., Ghosh, A.: 'Frequency Support for Remote Microgrid Systems With Intermittent Distributed Energy Resources -',IEEE Syst. J., 2018, 10, (3), pp. 2760-2771.

[16] Laghari, J.A., Mokhlis, H., Karimi, M., Halim, A. Bakar, A., Mohamad, H.: 'A New Under-Frequency Load Shedding Technique Based on Combination of Fixed and Random Priority of Loads for Smart Grid Applications',IEEE Trans. Power Syst., 2015, 30, (5), pp. 2507-2515.

[17] Oliveira, D.Q., Zambroni de Souza, A.C., Santos, M. V., Almeida, A.B., Lopes, B.I.L., Savedra, O.R.: 'A Fuzzy-Based Approach for Microgrids Islanded Operation',Electr. Power Syst. Res., 2017, 149, (1), pp. 178-189.

[18] Verayiah, R., Mohamed, A., Shareef, H., Abidin, I.Z. 'Review of Under-voltage Load Shedding Schemes in Power System Operation',Prz. Elektrotechniczny, 2014, 90, (7), pp. 99-103.

[19] Zambroni de Souza, A.C., Lima Lopes, B.I.: 'QuasiDynamic Model and Strategy for Control Actions',Electr. Power Components Syst., 2005, 33, (9), pp. 1057-1070.

[20] de Nadai N., B., Zambroni de Souza, A.C., de Carvalho Costa, J.G., Marujo, D., Portelinha Jr., F.M., Oliveira, D.Q.: 'A Monte Carlo Simulation Approach to Determine Critical Buses in Islanded Microgrids', in '5th IET International Conference on Renewable Power Generation (RPG) 2016' (IET, 2016), pp. 2-7

[21] Sortomme, E., Venkata, S.S., Mitra, J.: 'Microgrid protection using communication-assisted digital relays',IEEE Trans. Power Deliv., 2010, 25, (4), pp. 2789-2796.

[22] Nourollah, S., Pirayesh, A., Aminifar, F.: 'A Combinational Scheme for Voltage and Frequency Recovery in an Islanded Distribution System', IET Gener. Transm. Distrib., 2016, 10, (12), pp. 2899- 
2906.

[23] Hoseinzadeh, B., Faria Da Silva, F.M., Bak, C.L.: 'Adaptive Tuning of Frequency Thresholds Using Voltage Drop Data in Decentralized Load Shedding',IEEE Trans. Power Syst., 2015, 30, (4), pp. 2055-2062.

[24] Majumder, R.: 'Some aspects of stability in microgrids',IEEE Trans. Power Syst., 2013, 28, (3), pp. 3243-3252.

[25] ANEEL: 'Resolução Normativa $\mathrm{N}^{\circ} 414$ ', ((in portuguese), 2011)

[26] Zambroni de Souza, A.C., Santos, M., Castilla, M., Miret, J., García de Vicuña, L., Marujo, D.: 'Voltage security in AC microgrids: a power flow-based approach considering droop- controlled inverters',IET Renew. Power Gener., 2015, 9, (8), pp. 954-960.

[27] Sarmiento, J.E., Carreno, E.M., Zambroni de Souza, A.C.: 'Modeling Inverters with Volt-Var Functions in Grid-Connected Mode and Droop Control Method in Islanded Mode',Electr. Power Syst. Res., 2018, 155, pp. 265-273.

[28] Rese, L., Simões Costa, A., Silveira e Silva, A.: 'A Modified Load Flow Algorithm for Microgrids Operating in Islanded Mode', in 'Innovative Smart Grid Technologies Latin America (ISGT LA)' (2013), pp. 1-7

[29] Mumtaz, F., Member, S., Syed, M.H., et al.: 'A Novel Approach to Solve Power Flow for Islanded Microgrids Using Modified Newton Raphson With Droop Control of DG',IEEE Trans. Sustain. Energy, 2016, 7, (2), pp. 493-503.

[30] ANEEL: 'PRODIST(Procedimentos de Distribuição) Módulo 8 - Qualidade da Energia Elétrica', (in portuguese, 2016)

[31] Yang, X., Zong, X., Yang, F., Zang, H.Y.: 'A research on droop control strategy and simulation for the micro-grid',2011 Int. Conf. Electr. Control Eng. ICECE 2011 - Proc., 2011, pp. 5695-5700.

[32] Shongwe, S., Hanif, M.: 'Comparative Analysis of Different Single-Diode PV Modeling Methods',IEEE J. Photovoltaics, 2015, 5, (3), pp. 938-946.

[33] Billinton, R., Bai, G.: 'Generating Capacity Adequacy Associated with Wind Energy',IEEE Trans. Energy Convers., 2004, 19, (3), pp. 641-646.

[34] Lagace, P.J., Vuong, M.H., Kamwa, I.: 'Improving Power Flow Convergence by Newton Raphson with a Levenberg-Marquardt Method', in IEEE (Ed.): 'IEEE Power and Energy Society 2008 General Meeting: Conversion and Delivery of Electrical Energy in the 21st Century, PES' (2008), pp. 1-6

[35] Mohn, F.W., Souza, A.C.Z. De: 'Tracing PV and QV Curves With the Help of a CRIC Continuation Method',IEEE Trans. Power Deliv., 2006, 21, (3), pp. $1115-1122$.

[36] Marujo, D., Zambroni de Souza, A.C., Lima Lopes, B.I., Santos, M. V., Lo, K.L.: 'On Control Actions Effects by Using QV Curves',IEEE Trans. Power Syst., 2015, 30, (3), pp. 1298-1305.

[37] Moura, R.S., Zambroni de Souza, A.C., Lima Lopes, B.I., Mohn, F.W.: 'Effects of QV curves in the dynamic behaviour of power systems',IET Gener. Transm. Distrib., 2016, 10, (12), pp. 2861-2870.
[38] Santos, M.V., Zambroni de Souza, A.C., Lima Lopes, B.I., Marujo, D.: 'Secondary Voltage Control System Based on Fuzzy Logic',Electr. Power Syst. Res., 2015, 119, (1), pp. 377-384.

[39] Billinton, R., Allan, R.N.: 'Reliability Evaluation of Engineering Systems: Concepts and Techniques', (Plenum Press, 1992, Second)

[40] Baughman, M.L., Liu, C.-C., Dugan, R.C.: 'IEEE 37 Node Test Feeder', (1921)

[41] Mwakabuta, N., Sekar, A.: 'Comparative Study of the IEEE 34 Node Test Feeder Under Practical Simplifications', in '2007 39th North American Power Symposium, NAPS' (2007), pp. 484-491

[42] Oliveira, T.E.C. De, Carvalho, P.M.S., Ribeiro, P.F., Bonatto, B.D.: 'PV Hosting Capacity Dependence on Harmonic Voltage Distortion in Low-Voltage Grids : Model',Energies, 2018, 11, p. 465. 